\title{
INFORMACIONISTA DE PESQUISA: OPORTUNIDADES PARA BIBLIOTECÁRIOS NA ERA DO BIG DATA.
}

\section{RESEARCH INFORMATIONIST: OPPORTUNITIES FOR LIBRARIANS IN THE BIG DATA ERA}

\author{
Dayanne da Silva Prudencioa \\ Jorge Calmon de Almeida Biolchini ${ }^{b}$
}

\begin{abstract}
RESUMO
Introdução: apresenta oportunidades de atuação do bibliotecário de ciências da saúde como informacionista de pesquisa, em resposta às novas exigências de divulgação de dados de pesquisa requeridas por agências de fomento e editoras científicas. Objetivo: como objetivo principal está o debate de como este profissional pode contribuir com o desenvolvimento de estratégias para busca e divulgação de dados na área de Ciências da Saúde no contexto brasileiro. Metodologia: utiliza uma quasi-systematic review como método de pesquisa para apresentar um panorama histórico da ocupação, examinar o perfil profissional em termos de competências e habilidades necessárias a atuação no mundo do trabalho e ilustra algumas experiências profissionais documentadas na literatura internacional que sugerem uma trilha de boas práticas que podem ser aplicadas ao contexto nacional. Trata-se de pesquisa exploratória-descritiva, com abordagem qualitativa para análise dos dados e demonstração dos resultados. Resultados: evidencia que estes profissionais atuam como mediadores entre equipes clínicas e a informação especializada e atualizada. Além disso, operam em todas as etapas do processo de pesquisa, provendo orientações sobre formatos, fontes de publicação, disposições relacionadas ao direito de autor e assessoram todo o processo editorial. Entretanto, sua principal missão está relacionada a melhoraria da gestão de dados de pesquisa. Conclusão: conclui-se que a atuação do informacionista de pesquisa permite que o bibliotecário seja reconhecido como coprodutor de conhecimento em saúde e que efetivamente cumpra papéis mais ativos no desenvolvimento científico.
\end{abstract}

Descritores: Bibliotecário. Informacionista de pesquisa. Informacionista. Gestão de Dados. Profissional da informação.

\footnotetext{
a Doutoranda pelo Programa de Pós-Graduação em Ciência da Informação da Universidade Federal do Rio de Janeiro (PPGCI-UFRJ) em convênio com o nstituto Brasileiro de Informação em Ciência e Tecnologia (IBICT) e Professora Assistente do Curso de Biblioteconomia da Universidade Federal do Rio de Janeiro (UFRJ). E-mail: dayanneprudencio@gmail.com.

b Pesquisador do Instituto Brasileiro de Informação em Ciência e Tecnologia - IBICT - do Ministério da Ciência, Tecnologia, Inovações e Comunicações - MCTIC. E-mail: jorge.biolchini@gmail.com
} 


\section{INTRODUÇÃO}

Este artigo é, na realidade, um recorte de uma pesquisa mais ampla realizada a nível de doutoramento, cuja questão discute como os processos de aprendizagem influenciam no desenvolvimento das práticas informacionais dos bibliotecários que atuam na área de saúde no contexto brasileiro.

Especificamente nesta comunicação, apresentamos e exploramos as oportunidades de atuação do bibliotecário de ciências da saúde como informacionista de pesquisa. Um perfil profissional, ainda emergente, tanto no cenário nacional quanto internacional, e que entre fatores, surge para contribuir no processo de resposta às novas exigências de divulgação de dados de pesquisa requeridas por agências de fomento e editoras científicas. Na mesma linha, coopera para o atendimento dos princípios do accountability previsto por Gibbons et al. (1994).

Nessa perspectiva, entende-se que as práticas informacionais realizadas por estes profissionais, são manifestações dos novos processos de pesquisa da sociedade contemporânea, previstos na obra The New Production of Knowledge (Ibidem). Portanto, nessa orientação à aplicação sustentada no modo 2 de Gibbons, há uma alteração no escopo do fazer científico e reforçase a necessidade de diminuir a lacuna existente entre o novo conhecimento e a sua utilização para a melhoria da vida dos cidadãos da sociedade civil. Isso significa que o contexto de aplicação do fazer científico necessita absorver 0 social.

Observa-se o fim da centralidade da universidade e dos institutos de pesquisa como os únicos "lugares" produtores de ciência e conhecimento. Trata-se de uma rede de parcerias de pesquisa onde entram muitos outros atores. Nessa perspectiva, o modo 2, mais do que significar uma nova forma de produção do conhecimento, irá orientar como esse conhecimento é produzido, organizado, disseminado e aplicado. Essas novas facetas produzem terreno fértil para o questionamento acerca do papel de protagonista exclusivo da universidade e das outras instituições de pesquisa e, consequentemente, emerge a necessidade de prestação de contas (accountability) e de controle de 
qualidade (quality control) (GONZÁLEZ DE GÓMEZ, 2003, p. 71).

$O$ processo de accountability aqui é compreendido para além de uma mera prestação de contas, trata-se do resultado de um processo relacionado à mensuração da eficiência universitária em produzir conhecimentos que sejam relevantes ao saneamento dos problemas que a sociedade atravessa. Nesse sentido, cada vez menos há espaço e fomento à produção de conhecimentos desconectados da realidade social, de forma que as estruturas dos campos precisam e precisarão continuar justificando suas produções, produtos e aplicações. Essencialmente, trata-se de responder três questões: para quem, como e o quê (GONZÁLEZ DE GÓMEZ, 2003). O controle de qualidade, por sua vez, relaciona-se ao valor social agregado (Ibidem).

Sob tais circunstâncias, González de Gómez (2003) identifica que ocorrem processos de negociação de interesses e recursos e que as relações entre trabalho e pesquisa também sofrem modificações. Portanto, verifica-se a necessidade de novas competências e conhecimentos para atuação no ciclo de pesquisa, reorganização das posições dos agentes, bem como 0 desenvolvimento de novos perfis profissionais.

Nesta direção, inferimos que os diferentes desafios existentes no campo da pesquisa aplicada de saúde, no contexto nacional, podem beneficiar-se dos múltiplos saberes, habilidades, competências procedimentais ${ }^{1}$, atitudinais ${ }^{2}$, gerais $^{3}$ e específicas ${ }^{4}$ dos informacionistas de pesquisa. Ou seja, esses profissionais podem contribuir no entendimento das necessidades informacionais, localização, recuperação, filtragem e armazenamento dos dados e informações que permitem o desenvolvimento empírico e teórico do campo da saúde no Brasil, bem como, no aperfeiçoamento do processo de

1 Tipo de conteúdo de aprendizagem prático que consiste em processos ou ações cuja aprendizagem requer um modelo prévio e uma exercitação posterior. (ZABALA; ARNAU, 2010, p. 190).

2 Tipo de conteúdo de aprendizagem que se enquadra na forma de ser da pessoa e cuja aprendizagem requer a experienciação de situações nas quais se deva agir de forma real para solucioná-las (ZABALA; ARNAU, 2010, p. 190).

${ }^{3}$ Aquelas que atendem diferentes perspectivas de fazeres biblioteconômicos que circundam as práticas de trabalho (PRUDENCIO, 2015)

4 [...] intervenção eficaz diante de uma situação problema por meio de ações nas quais se mobilizem, ao mesmo tempo e de maneira inter-relacionada, componentes atitudinais, procedimentais e conceituais. (ZABALA; ARNAU, 2010, p. 36). 
tomada de decisão em relação ao sistema, área ou necessidade contextualizada no campo.

Abalizado no exposto, essa pesquisa tem como objetivo central, discutir como o informacionista de pesquisa pode contribuir com o desenvolvimento de estratégias para busca e divulgação de dados de pesquisa na área de Ciências da Saúde no Brasil?

Subsequente, delineou-se os seguintes objetivos específicos: a) apresentar um panorama histórico do termo informacionista de pesquisa; b) examinar esse perfil profissional, em termos de competências e habilidades; c) analisa as experiências profissionais documentadas na literatura internacional, que guardadas as diferenças sociais, econômicas, políticas e regulatórios, sugerem uma trilha de boas práticas que podem ser seguidas, de algum modo, no contexto nacional.

O trabalho inicia-se com a contextualização do tema. Em seguida, apresentamos nossos procedimentos metodológicos. Posteriormente, delineamos nossa abordagem teórica para contextualizar a temática investigada e análise realizada. Subsequentemente, relacionamos a atuação dos bibliotecários médicos e clínicos brasileiros aos programas de informacionistas de pesquisa discutidos na literatura internacional. A fim e a cabo, sugerimos ações para que esta profissão possa ser desenvolvida no contexto brasileiro.

\section{PROCEDIMENTOS METODOLÓGICOS}

Este estudo realizou uma quasi-systematic review (TRAVASSOS et al., 2008), método que, segundo os autores, deriva da revisão sistemática de literatura, seguindo o mesmo rigor para as etapas metodológicas e desenvolvendo um protocolo de pesquisa, mas não adotando uma checagem cruzada com a utilização de mais de um revisor para comparação e maior consistência de julgamento.

Cumpre informar que esta metodologia surgiu no campo da medicina e posteriormente foi adaptada a área de Engenharia de Software, contudo nos 
últimos anos tem recebido maior atenção no campo das ciências sociais aplicadas, tais como a Ciência da Informação.

Utilizando-se desta metodologia foi possível compreender 0 posicionamento de temáticas correlatas à nossa pesquisa, sendo elas: modelos de aprendizagem; formação do bibliotecário; espaços de trabalho; e competências do bibliotecário - sempre com encaminhamento ao campo da saúde.

De acordo com Biolchini et al. (2005) na aplicação desta metodologia é possível auferir mais rigor nos processos de coleta, avaliação e análise da literatura disponível acerca de dado tema ou questão de pesquisa. Para tanto, - pesquisador deve utilizar-se de rígidas etapas metodológicas, que basicamente orientam três fases: planejamento, execução e relatórios/publicação de resultados - cada uma destas são descritas a seguir. Ainda de acordo com Biolchini et al. (2005) essa disposição aufere publicações de alto valor científico e resultados confiáveis, inclusive no que tange a reprodutibilidade dos dados.

Seguindo etapas postuladas pela abordagem, iniciamos a fase de planejamento com a definição da questão de pesquisa, formulação do protocolo de pesquisa e acepção das expressões de busca.

Subsequentemente realizamos a definição dos quatro componentes da estratégia PICO (Pai et al. 2004), vide tabela 1 abaixo.

Tabela 1 - Descrição da Estratégia PICO

\begin{tabular}{l|l|l} 
ACRÔNIMO & \multicolumn{1}{|c|}{ DEFINIÇÃO } & \multicolumn{1}{c}{$\begin{array}{l}\text { DESCRIÇÃO } \\
\text { Artigos de periódicos e anais de } \\
\text { Pongresso relacionados com } \\
\text { informacionista de pesquisa. }\end{array}$} \\
\hline $\mathbf{I}$ & $\begin{array}{l}\text { Paciente ou } \\
\text { População }\end{array}$ & $\begin{array}{l}\text { Atuação do informacionista de } \\
\text { pesquisa com a gestão de dados }\end{array}$ \\
\hline $\mathbf{C}$ & Intervenção & $\begin{array}{l}\text { Bibliotecários médicos e } \\
\text { Bibliotecários clínicos em suas } \\
\text { atuações }\end{array}$ \\
\hline $\mathbf{O}$ & $\begin{array}{l}\text { Controle ou } \\
\text { Comparação }\end{array}$ & $\begin{array}{l}\text { Atividades e contribuição do } \\
\text { informacionista com a gestão de } \\
\text { dados de pesquisa }\end{array}$ \\
\hline
\end{tabular}

Fonte: Dados da pesquisa (2019). 
A revisão inclui todos os estudos retornados pelo protocolo que cumpriu pelo menos um dos seguintes critérios de inclusão abaixo.

- estudos em português, inglês e espanhol;

- documento publicados em periódicos indexados nas bases de dados BRAPCI, LISA, PUbMED, LILACS;

- documento publicados no portal da Biblioteca Virtual em Saúde:BVS (BIREME);

- restringiu-se a um tipo profissional específico: os informacionistas de pesquisa e sua atuação profissional. Ou seja, ainda que o trabalho representasse ações próximas às típicas de um informacionista de pesquisa, estes não foram considerados, pois não foram classificadas por seus autores como desta ocupação profissional;

- documentos devem discutir oportunidades e desafios para os informacionistas de pesquisa;

- os documentos devem relatar experiências de organizações ou profissionais que implementaram práticas de informacionistas de pesquisa em suas equipes.

Como critérios de exclusão, foram assumidos:

- idiomas diferentes dos já mencionados;

- indexados em bases de dados e portal distinto dos anunciadas;

- materiais que não fossem revisados pelos pares;

- estudos que não tratassem especificamente de atividades relacionadas ao escopo de atuação do informacionista de pesquisa.

Entretanto, na seção 4, que trata da atuação do bibliotecário médico e bibliotecário clínico no Brasil, fazemos uma comparação com as competências e habilidades elencadas na literatura para o informacionista.

$\mathrm{Na}$ fase de execução, procedemos a busca nas bases de dados indicadas acima e utilizou-se as seguintes palavras expressões-chave: researchers and data; research informationist; research data management $e$ data librarian.

Aplicando os critérios de inclusão acima indicados, obtivemos os seguintes resultados constantes na tabela 2 . 
Tabela 2 - Seleção primária de materiais

\begin{tabular}{l|l|l|}
$\begin{array}{l}\text { BASE DE } \\
\text { DADOS E } \\
\text { PORTAL }\end{array}$ & QUANTIDADE & FILTROS \\
\hline PUBMED & 700 & $\begin{array}{l}\text { Título, palavra chave } \\
\text { e resumo }\end{array}$ \\
\hline $\begin{array}{l}\text { BIBLIOTECA } \\
\text { VIRTUAL EM }\end{array}$ & 105 & $\begin{array}{l}\text { Título, palavra chave } \\
\text { e resumo }\end{array}$ \\
$\begin{array}{l}\text { SAÚDE: } B V S \\
(B I R E M E)\end{array}$ & 403 & $\begin{array}{l}\text { Título, palavra chave } \\
\text { e resumo }\end{array}$ \\
\hline LISA & 3 & $\begin{array}{l}\text { Título, palavra chave } \\
\text { e resumo }\end{array}$ \\
\hline BRAPCI & 8 & $\begin{array}{l}\text { Título, palavra chave } \\
\text { e resumo }\end{array}$ \\
\hline LILACS & Total: 1209 &
\end{tabular}

Fonte: Dados da pesquisa (2019).

Tendo cumprido a seleção inicial dos textos, procede-se seu registro no software Zotero e eliminamos as duplicadas que representaram $69 \%$ dos dados recolhidos. Tal repetição é reflexo da sobreposição entre documentos indexados em diferentes bases de dados. Em seguida recorreu-se ao método de análise de assunto que, segundo Naves (2001, p. 192), é "[...] o processo de ler um documento para extrair conceitos que traduzam a sua essência [...]".

Desta forma, examinou-se os resumos dos materiais visando selecionar itens relacionados a nossa questão de pesquisa. Quando ao final da leitura do resumo não ficou claro se o estudo correspondia aos nossos critérios de inclusão, procedemos a uma leitura da introdução.

Tendo cumprindo estas etapas, o quantitativo final é evidenciado na tabela 3 a seguir.

Tabela 3 - Seleção final de materiais

\begin{tabular}{l|l|} 
BASE DE DADOS E PORTAL & QUANTIDADE \\
\hline PUBMED & 74 \\
\hline BIBLIOTECA VIRTUAL EM SAÚDE: BVS & 33 \\
\hline LISA & 108 \\
\hline BRAPCI & 0 \\
\hline LILACS & 2 \\
& Total: 217
\end{tabular}

Fonte: Dados da pesquisa (2019). 
Portanto, 217 foi o quantitativo final que operou como itens de análise de nossa pesquisa.

Tendo apresentado as atividades relativas as fases de planejamento e execução do protocolo de pesquisa, classificamos a pesquisa como do tipo bibliográfico, quanto aos meios; exploratório-descritivo, no que se refere ao seu objetivo. Além disso, utiliza-se abordagem qualitativa para análise dos dados e demonstração dos resultados.

$\mathrm{Na}$ seção a seguir, apresentamos os resultados relacionados a análise dos corpus de pesquisa selecionado e que atende a questão de pesquisa que norteia nosso estudo.

\section{INFORMACIONISTA E INFORMACIONISTA DE PESQUISA: NOVAS OCUPAÇÕES E PRÁTICAS INFORMACIONAIS}

A primeira vez que o termo informacionista surge na literatura foi no artigo de Frank Davidoff e Valerie Florance, datado de 2000 e publicado no Annals of Internal Medicine. Neste estudo, os autores relacionam três categorizações profissionais de bibliotecários no campo da saúde, a saber: bibliotecário médico, bibliotecário clínico e informacionista.

Essa última categoria é colocada como uma evolução de bibliotecário clínico e se contextualiza com os papéis assumidos no contexto do princípio da Medicina Baseada em Evidência, no qual cada vez mais os profissionais de informação desempenham funções relevantes no processo de pesquisa de evidências científicas. Neste sentido, o conceito (e termo) informacionista é mais abrangente e coerente com as mudanças no perfil profissional do século $\mathrm{XXI}$.

Galvão e Leite (2008, p. 186), define o profissional como:

[...] aquele que possui conhecimento tanto no campo da organização e representação da informação quanto no próprio campo da saúde, e que compreende mais pormenorizadamente a terminologia do campo da saúde e contribui de forma ativa, seja na decisão médica, seja na construção do conhecimento médico [...].

Portanto, o informacionista trabalha como mediador entre as equipes 
clínicas e a informação especializada e atualizada, buscando as melhores evidências científicas a serem tratadas pelo corpo clínico, analisando os dados e aplicando em pesquisas de acordo com os casos (DAVIDOFF; FLORANCE, 2000).

Trata-se, portanto, de um especialista na resolução de problemas informacionais relacionados a temática em que atua. Sendo assim, atua sobre um grande volume de literatura e objetiva validar as informações relevantes e válidas para aquele contexto especial. (HERSH, 2002).

Rankin, Grefsheim e Canto (2008) relatam que a definição do informacionista continua a ser redefinida, conforme Federer (2013) tanto na Biblioteconomia quanto na literatura clínica, mas que sua reponsabilidade continua sendo a de fornecer serviços de informação altamente especializados para a equipe médica em que está inserido e demais profissionais a qual presta serviços.

O desenvolvimento desta ocupação decorre entre outros motivos da falta de tempo que os médicos e demais profissionais da saúde têm para se dedicar a leitura - ainda que esta seja necessária para uma eficiente e eficaz prática clínica e de pesquisa e pela necessidade de síntese das evidências científicas disponíveis (DAVIDOFF; FLORANCE, 2000). Neste sentido, o informacionista opera como um mediador entre a evidência disponível e a aplicação em contexto.

Sua atuação é assumida pelos órgãos de saúde e informação americanos como estratégica (COOPER, 2011). Tanto que a National Library of Medicine (NLM) esteve envolvida desde o início da criação do cargo de informacionista e, inclusive, foi a instituição responsável por estabelecer atributos e habilidades necessárias ao exercício do cargo, o que se deu durante uma conferência ocorrida no ano de 2002, em parceria com a Medical Library Association (DEARDORFF; FLORANCE; VANBIERVLIET, 2016).

Esta conferência resultou em um programa oficial de formação de informacionistas e, de acordo com Florance (2013), entre 2003 e 2008 este programa formou nove informacionistas de oito estados americanos. 0 programa desenvolveu suas atividades em quatro áreas consideradas 
prioritárias pelo sistema de saúde estadunidense, sendo elas: pesquisa clínica geral, clínica biomédica, saúde pública e saúde do consumidor.

A segunda edição do programa iniciou-se em 2010 e vigorou até 2016. Entretanto, o foco da segunda edição não foi a formação e sim a atuação em pesquisas relacionadas a temas como hidratação da córnea, rastreio de câncer de mama, redução de tabagismo, entre outros. Essa atuação se relacionou mais com o que se entende como escopo do informacionista de pesquisa (FLORANCE, 2013).

Não há consenso na literatura acerca dos conhecimentos, competências e habilidades para atuação no cargo. Em geral, a discordância concentra-se na obrigatoriedade de formação na área de saúde - tese defendida por Robison (2008). No entanto, para autores como Cataldo et al. (2006) e Fikar; Corral (2001), este aspecto é apenas desejável.

Em uma terceira abordagem, Federer (2013) defende que o informacionista é um profissional que atua diretamente no ambiente de trabalho (hospital, escritório, laboratório etc.) de seus clientes/usuários, e, portanto, suas práticas são situadas na aplicação do conhecimento em domínio. Sendo assim, argumenta que o mais importante é que este profissional detenha conhecimentos especializados no campo de prática em que atua. Para tanto sugere que estes sejam obtidos de distintas maneiras, não necessariamente com formação universitária.

O formato dessa formação também é alvo de divergência, Davidoff e Florance (2000), entendem que informacionistas deveriam ser formados a partir de um programa nacional com um currículo padronizado e credenciamento junto a órgãos formais. Byrd (2002) e Homan; McGowan (2002) defendem a qualificação em escolas formais e que isso deveria ser o pré-requisito para o exercício da profissão. Por outro lado, autores como Brown (2004) argumentam que a formação deveria ocorrer mediante treinamentos situados nas práticas de trabalho, autoaprendizagem e educação continuada. Aproximando-se do postulado por Federer (2013). Cumpre informar que NIH Library continua a oferecer um programa de formação de informacionista.

Nestes programas são ensinados sobre o uso de programas de 
gerenciamento de informações, como EndNote e Mendeley, softwares de análises bibliométricas e mensuração do impacto de pesquisa, análise de estatística de publicação, bases de conhecimentos e dados, visualização de dados, ferramentas de compreensão e significado biológico. Também há instruções acerca de treinamentos e consultoria informacional. (NATIONAL INSTITUTES OF HEALTH, 2019b).

A partir do exame da literatura selecionada, verificamos que apenas um dos nove informacionistas formados no programa da NLM, não possuía formação inicial na área da saúde. O fato era previsto, haja vista que naquele país a formação biblioteconômica ocorre na pós-graduação, neste sentido, é possível haver diferentes profissionais com pós-graduação em Biblioteconomia ou Ciência da Informação.

Cumpre ainda informar, que há cursos de pós-graduação na área de informação, com ênfase em áreas estratégicas como a da saúde, tal como ocorre como o da Universidade de Michigan. Nessa perspectiva, há indícios que precisam ser confirmados em pesquisas futuras, que profissionais de informação formados neste modelo possuem uma aprendizagem mais efetiva às suas práticas como informacionistas.

No que tange as competências necessárias a este profissional, temos na uma revisão sistemática com cobertura de 2000 a 2006 de autoria de Rankin, Grefsheim e Canto (2008) importantes contribuições e síntese, conforme constam no quadro 1.

Quadro 1 - Competências desejáveis ao profissional Informacionista

\begin{tabular}{|c|c|c|}
\hline Habilidades Pessoais & Conhecimento & $\begin{array}{l}\text { Habilidades } \\
\text { Funcionais }\end{array}$ \\
\hline $\begin{array}{l}\text { Comunicação; } \\
\text { Profissionalismo; } \\
\text { Aprendizagem a longo da } \\
\text { vida; } \\
\text { Garantia de qualidade; } \\
\text { Pro atividade; }\end{array}$ & $\begin{array}{l}\text { Domínio clínico e } \\
\text { biblioteconômico; } \\
\text { Ambiente informacional; } \\
\text { Projetos de pesquisa e } \\
\text { análise; } \\
\text { Tecnologia; }\end{array}$ & $\begin{array}{l}\text { Pesquisar e localizar } \\
\text { informações; } \\
\text { Avaliar criticamente; } \\
\text { Sintetizar a } \\
\text { informação; } \\
\text { Gerenciamento e } \\
\text { organização de }\end{array}$ \\
\hline
\end{tabular}




\begin{tabular}{|l|l|l|}
\hline Liderança; & Organizacional; & informações; \\
$\begin{array}{l}\text { Serviço de atendimento } \\
\text { ao cliente; }\end{array}$ & $\begin{array}{l}\text { Disciplinas relacionadas } \\
\text { a política e regulação, } \\
\text { conceitos básicos de } \\
\text { Empreendedorina, princípios da }\end{array}$ & $\begin{array}{l}\text { Gerenciamento de } \\
\text { projetos; }\end{array}$ \\
$\begin{array}{l}\text { epidemiologia clínica, } \\
\text { bioestatística, avaliação do } \\
\text { crítica da literatura, } \\
\text { gerenciamento de } \\
\text { informações, entre outras }\end{array}$ & conhecimento; \\
Educação; \\
Informática aplicada \\
\hline
\end{tabular}

Fonte: RANKIN; GREFSHEIM; CANTO (2008)

Deardorff; Florance e VanBiervliet (2016), Grefsheim et al. (2010), Cooper (2011) e Montano (2016), são alguns dos autores que investigaram os domínios de aplicação destas competências. Desta forma, indicam que as principais atividades relacionadas à atuação dos informacionistas são:

- Captura, gerenciamento, armazenamento, organização de dados;

- Análise de dados de biociência;

- Avaliação e síntese da literatura com responsabilidade direta pela qualidade dos resultados;

- Treinamento de usuários sobre os princípios da Medicina Baseada em Evidência;

- Gestão do conhecimento;

- Preparação de artigos e coautoria em publicações;

- Facilitação em demandas de colaborações na instituição e externamente.

Cumpre informar, que as ações acima mencionadas são um recorte de acordo com as indicações mais recorrentes e, todavia, Shipman; Homan (2003) e Allee et al. (2014) ressaltam que o papel do informacionista altera-se mediante o contexto de atuação. Ou seja, conforme as necessidades da comunidade usuária de seus serviços, direcionamento de atuação, equipe gestora, tecnologia e recursos informacionais disponíveis.

Os estudos recuperados nesta pesquisa apontam que dezenove anos após a formação do primeiro grupo de informacionistas, a ocupação segue em 
franco desenvolvimento, tem expandido seu alcance e aperfeiçoado suas práticas. Diversos relatos de experiência foram analisados, e, evidenciam a contribuição destes profissionais com o fomento da literatura profissional, sociabilização dos conhecimentos adquiridos e um aporte ao desenvolvimento empírico da área.

Um dos exemplos desta expansão, é a especialização da ocupação com orientação às atividades de pesquisa, desta forma, haveria o informacionista clínico (BROWN, 2004) e o informacionista de pesquisa (FEDERER, 2013), categoria profissional que discutimos a seguir.

\subsection{Informacionista de PEsquisa}

A indicação da profissão surge pela primeira vez em um anúncio da National Library of Medicine (NLM), no ano de 2011, sobre uma oportunidade de financiamento para atuação de especialista em informação em equipes de pesquisa visando melhorar a captura, armazenamento, organização, gerenciamento, integração, apresentação e disseminação de informações e dados de pesquisa biomédica (FEDERER, 2013). Contudo, o primeiro artigo sobre a temática é publicado apenas em 2013 no Journal of the Medical Library Association (JMLA), e já apresenta algumas contribuições da ocupação ao processo de pesquisa.

O informacionista de pesquisa trabalha com equipes de investigação em cada etapa do processo de pesquisa, desde a definição da questão de pesquisa até sua publicação final. Inclusive, provê orientações sobre formatos, fontes de publicação e direito de autor e assessora todo o processo editorial. Entretanto, sua principal missão é melhorar a gestão de dados de pesquisa (FEDERER, 2013, p. 297).

Segundo Deardorff; Florance e VanBiervliet (2016), o informacionista de pesquisa é um especialista em informação, geralmente bibliotecário de ciências da saúde, com formação em pós-graduação e experiência prática em áreas relacionadas a ciências biomédicas e biológicas e/ou informática.

Federer (2013), assinala que a ocupação pode especificar a atuação 
conforme o campo, como: informacionistas biomédicos que trabalham com biologia e pesquisadores de bioinformática, informacionistas de saúde pública, informacionistas de saúde dos consumidores e informacionistas de investigação oncológica.

Cumpre informar que na literatura consultada há indicação de outras nomenclaturas atribuídas aos profissionais que atuam com práticas típicas de informacionistas de pesquisa, tais como: consultor de dados, bibliotecário de dados, agente de dados, consultor de gerenciamento de dados, curador de dados, oficial de dados, cientista de dados e entre outros. (KOLTAY, 2017). Contudo, é preciso esclarecer que o informacionista de pesquisa vai além do papel de gestor de dados, é também um especialista em pesquisa em dada área do conhecimento.

O surgimento da ocupação e seu desenvolvimento, decorre da insuficiência de conhecimentos teóricos e práticos que a maioria dos cientistas possuem para lidar com a avaliação de grandes conjuntos de dados, processos automatizados de mineração, verificação de autenticidade e das fontes disponíveis para captura de dados. (GIARLO, 2013; KOLTAY, 2017).

As principais competências para atuação como informacionista de pesquisa estão em torno do ciclo de vida de pesquisa, sendo elas: planejamento, coleta, gerenciamento, compartilhamento e publicação. (FEDERER, 2012). Além disso, auxiliam os pesquisadores a localizar dados sobre drogas, patentes, legislação, recursos de transferência e tecnologia aplicada a ciências biomédicas. (NATIONAL INSTITUTES OF HEALTH, 2019b).

No que se refere à formação destes profissionais, ainda há pouca literatura publicada. Contudo, devido às atividades de análise de dados desempenhadas, recomenda-se que os informacionistas possuam conhecimento do assunto em que atuam, não sendo necessário, porém, um grau avançado (FEDERER, 2012; 2013).

$\mathrm{Na}$ mesma linha, são essenciais conhecimentos acerca dos distintos modelos de licenciamento de publicações, das fontes existentes para coleta e depósito de dados, diferentes normas documentárias, mecanismos de 
preservação digital, softwares de análise de dados, política de financiadores de subsídios, regras aplicáveis as agências de fomento e familiaridade com as chamadas boas práticas científicas das comunidades de prática dos pesquisadores da área em que atuarão (FEDERER, 2013).

Tendo em vista que a atuação relacionada a gestão de dados é aspecto central das práticas dos informacionistas, a NIH Library desenvolve um programa de formação dedicada ao tópico, denominado Data Services Program. Neste há treinamentos sobre Gestão de dados, Visualização de dados, Análise de dados, R e RStudio e suporte para software estatístico. Os cursos são oferecidos presencialmente e por webinar. (NATIONAL INSTITUTES OF HEALTH, 2019a)

Outro ponto importante é que a NIH Library dispõe de infraestrutura física para que este informacionistas desenvolvam seu trabalho. Trata-se do Hub de Tecnologia da NIH Library. Neste espaço há ferramentas disponíveis, auxílio no uso de ferramentas, tutoriais e espaço de trabalho adequado. (NATIONAL INSTITUTES OF HEALTH, 2019a)

Cumpre também ressaltar, que desde 2014 a Association of College and Research Libraries (ACRL) através de seu Research Planning and Review Committee tem demonstrado preocupação com a formação de bibliotecários e outros profissionais de informação para atuação relacionada a partilha, gestão, curadoria, citação e gestão de dados. (KOLTAY, 2017). Neste sentido, o autor recomenda que estes profissionais invistam em formação continuada acerca do que denomina alfabetização de dados e que as instituições e órgãos de classe realizem treinamentos e eventos sobre o tema.

Há ainda no site da NIH Library, um portfólio que indica agências e profissionais referenciados para atuação como informacionistas. Atualmente há oito profissionais relacionados neste portal.

A atuação de bibliotecários como informacionistas de pesquisa tem exigido que este grupo invista em formação continuada, sobretudo em cursos relacionados à curadoria e gestão de dados da pesquisa e reutilização e pesquisa social (FEDERER, 2013). Também é comum a procura por cursos de bibliografia em Ciências da Saúde, fundamentos do campo em que atua, 
recuperação de informação, bioestatística e informática médica (DETLEFSEN, 2002).

A pesquisa em tela sugere que a formação destes profissionais ocorra em nível de especialização, sendo aberta inclusive para não bibliotecários. Contudo, sustentamos que especificamente no contexto da educação biblioteconômica praticada no Brasil - cuja base é generalista - ocorra maior ênfase em conteúdos relacionados ao ciclo de vida da pesquisa. Este deve ser discutido desde a formulação da questão até a publicação dos resultados, passando, evidentemente, por métodos e técnicas de pesquisa aplicada, fontes de informação, divulgação científica, técnicas de visualização e gerenciamento de dados, editoração e gestão de conteúdo. Entendemos que o trabalho de assessoria e de pesquisa ainda é um campo pouco explorado pelos bibliotecários brasileiros.

É importante destacar que profissionais mais experientes na função têm servido de instrutores aos novatos. Haja vista, que ainda não há um curso de pós-graduação ou formação complementar direcionado à formação exclusiva de informacionistas de pesquisa.

\subsection{EXPERIÊNCIAS INTERNACIONAIS: VIABILIDADE DA INCLUSÃO DE INFORMACIONISTAS}

Conforme já mencionado, foram recuperados artigos cuja centralidade são relatos de experiências acerca da incorporação de informacionistas em equipes multidisciplinares. Foram verificadas e/ou analisadas experiências na Austrália e Estados Unidos, Canadá, Irlanda, Nova Zelândia e Reino Unido respectivamente Sladek; Pinnock; Phillips (2004) e Federer (2013) e Tenopir, Birch e Allard (2012) e Corrall, Kennan e Afzal (2013).

Nossa análise verificou-se que esta atuação está relacionada a atividades de pesquisa, filtragem e fornecimento de dados e evidências que contribuam para a melhoria da qualidade das pesquisas médicas, sobretudo em temas estratégicos, como oncologia, saúde pública entre outros.

Averígua-se que há um reconhecimento que atualmente, produzir conhecimento e fazer ciência englobando apenas uma área do conhecimento é 
insuficiente frente às demandas e interesses operados no trinômio: empresas, governo e sociedade civil. Nesse sentido, tal como sustenta Gibbons et al. (1994) a saída encontrada pelo modelo produtivo foi utilizar o conceito de transdisciplinaridade.

A transdisciplinaridade emerge a partir de um contexto de aplicação particular e significa a mobilização de uma série de perspectivas teóricas e práticas metodológicas próprias para resolver problemas. A transdisciplinaridade ao contrário da inter e da multidisciplinaridade não necessariamente derivam de disciplinas. (GIBBONS et al., 1994, p. 168).

Em outras palavras, a transdisciplinaridade representa um esforço coletivo e consensual entre diferentes disciplinas para enfrentar e propor soluções a problemas. Essa diligência é desenvolvida e sustentada no domínio da aplicação. Outrossim, pode demandar a articulação de componentes teóricos e empíricos, bem como método de pesquisa e práticas distintas. Neste sentido, acredita-se que a prática informacional realizada por informacionistas é um exemplo de aplicação interdisciplinar.

Observa-se indícios Federer (2013) que a cooperação entre informacionista, médico e cientista permite um melhor atendimento ao paciente, pesquisa mais aprofundadas e melhoria dos programas educacionais de profissionais de saúde.

Há ainda o aperfeiçoamento de práticas de gestão de dados que permitem criar uma infraestrutura informacional de qualidade, permitindo que trabalhadores possam descobrir estratégias mais eficazes para prevenir, controlar e, agir terapeuticamente sobre doenças como o câncer.

Também foram declarados maiores investimentos na biblioteca que suporta o trabalho de pesquisa e qualificação dos profissionais envolvidos no serviço de referência. (MONTANO, 2016).

No exame e escopo da quasi-systematic review empreendida nesta pesquisa foram recuperados alguns materiais acerca da possível atuação do bibliotecário na gestão de dados (SILVA, 2016; COSTA; CUNHA, 2014 entre outros), porém nenhum relatando especificamente sobre esta atuação. Sendo assim, não se pode dizer que não ocorra, porém não foi anunciada na literatura científica e/ou profissional. 
Desta forma, encorajamos que os profissionais praticantes na área de ciência dos dados divulguem suas experiências, participem de eventos visando socializar seus conhecimentos e práticas, e, portanto, contribuam para o progresso empírico da área.

\section{CONTRIBUIÇÕES DO INFORMACIONISTA DE PESQUISA NA ERA DO BIG DATA}

Segundo Federer (2013, p. 298) a maioria dos grupos de pesquisadores contemplados com a inserção de um bibliotecário em suas equipes de pesquisa em virtude ao financiamento da NLM, possuíam uma consciência limitada dos tipos de serviços que os bibliotecários atuantes como informacionistas de pesquisa poderiam oferecer.

A autora segue seu relato apontando que para boa parte destes pesquisadores, estes profissionais seriam úteis apenas no serviço de referência e realização de levantamento bibliográficos. Contudo, com o início dos projetos de pesquisa, os bibliotecários identificaram limitações em interfaces do processo de pesquisa e então propuseram uma série de medidas práticas, tais como:

- Orientação sobre gerenciamento de dados e curadoria, padrões e metadados;

- Preservação e preparação de dados para partilha;

- Mecanismos de busca de meta-análises e revisões sistemáticas;

- Análise bibliométrica e análise de rede, para identificar colaboradores de pesquisa em potencial.

Desta forma, há uma desconstrução do perfil inicialmente concebido e instaura-se uma prática de interação e dupla contribuição. A mesma autora indica que, conforme foram sendo incorporados às comunidades de prática (WENGER, 1999), estes profissionais também forneciam orientações sobre as melhores práticas para a preservação de dados, como backup automatizado e redundância de armazenamento; adaptação para um padrão de metadados capaz de fornecer o contexto para os dados existentes e orientar futuros dados, 
bem como desenvolvimento de soluções para agregar dados de diferentes fontes e processo de coleta de dados mais eficiente.

Ainda como informacionistas de pesquisa, os bibliotecários atuam na localização de conjuntos de dados e, quando necessário, orientam as negociações do acesso a dados fechados, as consultas sobre acordos de partilha de dados e fornecem assistência com processos de dados desorganizados.

Outra interface do trabalho e seu perfil enquanto facilitador e educador de competências informacionais (UHSL, 1996 apud LAPPA, 2004), isso porque mesmo quando os conjuntos de dados são organizados de acordo com as melhores práticas, raramente vêm prontos para um pesquisador usar, o que permite os informacionistas de pesquisa atuarem em programas de formação de visualização de dados e desenvolver interfaces que facilitem esse trabalho, inclusive colocando proativamente os recursos e infraestrutura de bibliotecas a disposição dos pesquisadores.

Através de suas práticas profissionais, os informacionistas de pesquisa produzem conhecimento, criam métodos e redefine as relações entre profissional de informação e cientista.

Essas práticas têm trazido contribuições muito importantes sobre gestão de dados, tem auxiliado a compreensão sobre a importância de investimentos na criação e gestão de repositório de dados e de certa forma, aproximam o Brasil de uma agenda global sobre acesso aberto.

Sendo assim, verifica-se que as práticas dos informacionistas de pesquisa também cooperam para que a biblioteca aumente sua visibilidade e fundamente suas práticas para além de uma visão patrimonialista e gestora de acervos. Ou seja, o trabalho do informacionista dimensiona uma ação em que a pesquisa (como uma das maneiras de produzir conhecimento) seja a razão da biblioteca e não um dos itens de seu portfólio de serviços.

No atual contexto, onde cresce a passos largos a necessidade de publicização e depósito dos dados que subsidiam os processos de pesquisa, sugere-se que os informacionistas de pesquisa podem e continuarão a exercer importantes práticas, independentemente do porte da agência, editora ou 
instituição de pesquisa.

$\mathrm{Na}$ mesma linha, entendemos que a integração de um informacionista em equipes dedicadas a pesquisa permite melhorias nas ferramentas de estruturação e análise de dados, produzem impactos positivos na capacidade dos pesquisadores tomarem decisões baseadas em dados significativos e assim melhoram a qualidade das pesquisas em saúde.

No contexto brasileiro, merecem destaque as iniciativas empreendidas pela Fundação Oswaldo Cruz (Fiocruz), que, ao longo de 2018, desenvolveu diversas atividades para promover o debate sobre a gestão e a abertura de dados, visando contribuir com o saneamento de lacunas das políticas públicas brasileiras diante do emergente cenário global de pesquisa. Inclusive desenvolveu um evento intitulado "Abertura de Dados para pesquisa na Fiocruz: perspectivas de um novo paradigma da Ciência".

Neste encontro foram discutidos temas sobre uma política de abertura de dados, termos de referência e mapeamento de experiências em curso de abertura de dados para pesquisa. A instituição também possui um curso de atualização em Ciência de Dados Aplicada à Saúde em nível de pós-graduação lato sensu presencial oferecido pelo Instituto de Comunicação e Informação Científica e Tecnológica em Saúde (Icict/Fiocruz). Trata-se de uma importante opção de formação continuada para bibliotecários brasileiros interessados em atuar como informacionistas de pesquisa nos moldes do cenário internacional. Também é relevante citar que a Fiocruz desenvolveu uma plataforma de Ciência de Dados aplicada à Saúde ${ }^{5}$ e esta é uma interface para instituições de ensino, pesquisa e governo. A plataforma tem três pilares: análise de dados (indexação, extração e análise visual de dados); mineração de dados (conexão de dados da Plataforma via R Studio Server) e data Science Lab (inovação e aprendizagem colaborativa) (FUNDAÇÃO OSWALDO CRUZ, 2018).

O site da plataforma indica a equipe que atua na coordenação, desenvolvimento, referência e gestão de créditos. Verificamos que entre os 28 profissionais indicados, nenhum tem formação em nível de graduação em

\footnotetext{
${ }^{5}$ https://bigdata.icict.fiocruz.br/
} 
Biblioteconomia ou Ciência da informação indicada em seu currículo Lattes e apenas três profissionais têm formação em nível de doutorado em Informação e Comunicação em Saúde. Estes dados sugerem que os bibliotecários ainda têm sido pouco atuantes neste setor e os motivos para tal serão explorados em investigação futura, haja vista que não é escopo desta comunicação. Desse modo, os apontamentos apresentados até aqui evidenciam que há uma grande oportunidade para bibliotecários, que caberá a estes profissionais a responsabilidade por sua formação continuada (VALENTIM, 2002), mas que isso não exime as Escolas de Biblioteconomia, Ciência da Informação e órgãos de classe de empreender ações de formação em termos de conteúdos básicos e conteúdos profissionais essenciais para o desenvolvimento de competências e habilidades requeridas a este profissional.

\section{CONSIDERAÇÕES FINAIS}

Recomendamos que haja uma uniformidade de conteúdos e práticas em cursos de formação de informacionistas e que estes sejam oferecidos no Brasil. Atentamos para a relativa falta de experiência profissional neste tipo de entrega e que, portanto, é necessário qualificar estes profissionais antes de explorar um projeto piloto por exemplo.

Este estudo objetivou apresentar o conceito de informacionista de pesquisa, indicando suas competências, atribuições e, ao mesmo tempo, suas contribuições ao processo de gestão de dados na era do big data e consideramos que vide seção 3.1 foi cumprido. Na mesma linha, apresentou-se experiências profissionais documentadas na literatura internacional mediante seção 3.2.

Verificou que ainda será necessário o desenvolvimento de oportunidades de formação profissional capazes de atender às novas exigências de competência profissional requeridas pelo novo cenário de pesquisa mundial. Podemos concluir que os bibliotecários brasileiros devem buscar uma adequação de sua formação visando aproveitar as novas oportunidades que serão colocadas, inclusive para a maior valorização da 
profissão e criação de vínculo de confiança e parceria entre bibliotecários e pesquisadores.

Não se trata de ignorar que os bibliotecários já tem prestado relevantes serviços em atividades de pesquisa, sobretudo na construção de revisões sistemáticas de literatura, mas acreditamos que o formato de atuação do informacionista de pesquisa, nos moldes internacionais, é uma abordagem inovadora, com maior potencial de contribuição científica e maior maturidade instrucional para empreender ações de parceria em coautoria e comprometimento com o desenvolvimento teórico e empírico da área de informação e do cenário científico mundial.

A revisão empreendida evidenciou as possíveis contribuições do informacionista para o trabalho do pesquisador, a importância de uma eficiente gestão de dados de pesquisa e enfatizou a importância de formarmos profissionais para esta atuação.

\section{REFERÊNCIAS}

ALLEE, N. J.; BLUMENTHAL, J.; JORDAN, K.; LALLA, N.; LAUSENG, D.; RANA, G.; SAYLOR, K.; SONG, J. One institution's experience in transforming the health sciences library of the future. Medical Reference Services

Quarterly, [s.l.], v. 33, n. 1, p. 1- 16, jan. 2014. Disponível em: https://www.tandfonline.com/doi/abs/10.1080/02763869.2014.866444. Acesso em: 20 mar. 2019.

BIOLCHINI, J.; MIAN, P. G.; NATALI, A. C. C.; TRAVASSOS, G. H. Systematic Review in Software Engineering, Relatório Técnico ES, 2005. Disponível em: https://www.cos.ufrj.br/uploadfile/es67905.pdf. Acesso em: 15 jan. 2019.

BYRD, G. D. Can the profession of pharmacy serve as a model for health informationist professionals? Journal of the Medical Library Association (JMLA). v. 90, n. 1, p. 68-75. jan. 2002. Disponível em: https://www.ncbi.nlm.nih.gov/pubmed/11838462. Acesso em: 26 abr. 2019.

BROWN, H.-A. Clinical medical librarian to clinical informationist. Reference Services Review, West Yorkshire, v. 32, n. 1, p.45-49, 2004. Disponível em: https://www-

emeraldinsight.com.ez29.capes.proxy.ufrj.br/doi/pdfplus/10.1108/00907320410 519397. Acesso em: 06 abr. 2019. 
CATALDO, T. T.; TENNANT, M. R.; SHERWILL-NAVARRO, P.; JESANO, R. Subject specialization in a liaison librarian program. Journal of Medical Library Association, v. 94, n. 4, oct. 2006. p. 446-448. Disponível em: https://www.ncbi.nlm.nih.gov/pmc/articles/PMC1629419/pdf/i1536-5050-094-040446.pdf. Acesso em: 20 jun. 2019.

COOPER, D. Is the informationist a new role? A logic model analysis. Journal of Medical Library Association, v. 99, n. 3, p. 189-192, jul. 2011. Disponível em: https://www.ncbi.nlm.nih.gov/pmc/articles/PMC3133893. Acesso em: 18 mar. 2019.

CORRALL, S.; KENNAN, M. A., AFZAL, W. Bibliometrics and research data management services: Emerging trends in library support for research. Library Trends, v. 61, n. 3, p. 636-674, 2013. Disponível em: http://dscholarship.pitt.edu/18948/1/08_61_3_corrall_636-674.pdf. Acesso em: 20 jun. 2019.

COSTA, M. M.; CUNHA, M. B. O bibliotecário no tratamento de dados oriundos da e-Science: considerações iniciais. Perspectivas em Ciência da Informação, v. 19, n. 3, p. 189-206, 2014. Disponível em: http://hdl.handle.net/20.500.11959/brapci/37440. Acesso em: 02 maio 2019.

DAVIDOFF, F.; FLORANCE, V. The informationist: a new health profession? Annals of Internal Medicine, v. 132, n. 12, p. 996-998, 2000. Disponível em: https://www.ncbi.nlm.nih.gov/pubmed/10858185. Acesso em: 15 maio. 2019.

DEARDORFF, A.; FLORANCE, V.; VANBIERVLIET, A. Assessing the National Library of Medicine's Informationist Awards. Journal Escience Librarianship, v. 5, n. 1, out., 2016. Disponível em:

https://www.ncbi.nlm.nih.gov/pubmed/27818846 Acesso em: 15 nov. 2018.

DETLEFSEN, E. G. The education of informationists, from the perspective of a library and information sciences educator. Journal of Medical Library

Association, v. 90, n. 1, p. 59-67, 2002. Disponível em:

https://www.ncbi.nlm.nih.gov/pmc/articles/PMC64758. Acesso em: 15 fev. 2019.

FEDERER, L. New Roles for the 21st Century: The Research Informationist. In. ARL/CNI FALL FORUM, LIBRARY WORKFORCE FOR 21ST CENTURY RESEARCH LIBRARIESARL FALL FORUM, out. 2012. Disponível em: https://www.arl.org/wp-content/uploads/2012/10/ff12-federer.pdf. Acesso em: 02 jun. 2019.

FEDERER, L. The librarian as research informationist: a case study. Journal of Medical Library Association, v. 101, n. 4, p. 298-302, out., 2013. Disponível em: https://www.ncbi.nlm.nih.gov/pmc/articles/PMC3794685/. Acesso em: 30 jun. 2019.

FIKAR, C. R.; CORRAL, O. L. Non-librarian health professionals becoming librarians and information specialists: results of an Internet survey. Bull. Med. 
Libr. Assoc., Chicago, v. 89, n. 1, p. 59-67, jan. 2001. Disponível em: https://www.ncbi.nlm.nih.gov/pmc/articles/PMC31705/. Acesso em: 10 jun. 2019.

FLORANCE, V. Informationist careers for librarians: a brief history of NLM's Involvement. Journal of eScience Librarianship, v. 2, n. 1, 2013. Disponível em: http://dx.doi.org/10.7191/jeslib.2013.1040. Acesso em: 06 fev. 2019.

FUNDAÇÃO OSWALDO CRUZ. Ciência de dados: equipe. 2018. Disponível em: https://bigdata.icict.fiocruz.br/equipe . Acesso em: 10 jul. 2019.

GALVÃO, M. C. B.; LEITE, R. A. F. Do bibliotecário médico ao informacionista: traços semânticos de seus perfis e competências. Transinformação, v. 20, n. 2, p. 181-191, 2008. Disponível em:

https://brapci.inf.br/index.php/article/view/0000000488/932a9f8a54b00407376e b9c7141f82c2/. Acesso em: 16 maio 2019.

GIARLO, M. J. Academic libraries as quality hubs. Journal of Librarianship and Scholarly Communication, v. 1, n. 3, p. 1-10, 2013. Disponível em: https://jlsc-pub.org/articles/abstract/10.7710/2162-3309.1059/. Acesso em: 24 abr. 2019.

GIBBONS, M.; LIMOGES, C.; NOWOTNY, H.; SCHWARTZMAN, S.; SCOTT, P.; TROW, M. The new production of knowledge: the dynamics of science and research in contemporary societies. London, Thowsand Oaks, New Delhi: Sage Publications, 1994.

GONZALEZ DE GOMEZ, M. N. As relações entre ciência, Estado e sociedade: um domínio de visibilidade para as questões da informação. Ciência da Informação, Brasília, v. 32, n. 1, p. 60-76, jan./abr. 2003. Disponível em: http://www.scielo.br/pdf/ci/v32n1/15974.pdf. Acesso em: 02 maio 2019.

GREFSHEIM, S. F.; WHITMORE, S. C.; RAPP, B. A.; RANKIN, J. A.; ROBISON, R. R.; CANTO, C. C. The informationist: building evidence for an emerging health profession. Journal of Medical Library Association, v. 98, $\mathrm{n}$. 2, p. 147-156, abr. 2010. Disponível em:

https://www.ncbi.nlm.nih.gov/pmc/articles/PMC2859271/. Acesso em: 20 fev. 2019.

HERSH, W. Medical informatics education: An alternative pathway for training informationists. Journal of the Medical Library Association, v. 90, n. 1, p. 76-79, 2002. Disponível em: https://www.ncbi.nlm.nih.gov/pubmed/11838463. Acesso em: 25 mar. 2019.

HOMAN, J. M.; MCGOWAN, J. J. The Medical Library Association: promoting new roles for health information professionals. Journal Medical Library Association, v. 90, n. 1, p. 80-85, jan. 2002. Disponível em: https://www.ncbi.nlm.nih.gov/pmc/articles/PMC64761/. Acesso em: 24 abr. 2019. 
KOLTAY, T. Data literacy for researchers and data librarians. Journal of Librarianship and Information Science, v. 49, n. 1, p. 3-14, 2017. Disponível em:

https://journals.sagepub.com/doi/abs/10.1177/0961000615616450?journalCode =lisb. Acesso em: 20 maio 2019.

LAPPA, E. Clinical Librarianship (CL): A Historical Perspective. E-JASL, [s.I.], v. 5, n. 2-3, Sept. 2004. Disponível em:

http://southernlibrarianship.icaap.org/content/v05n02/lappa_e01.htm. Acesso em: 20 fev. 2019.

MONTANO, B. S J. Using the journal BMJ Case Reports to promote the publication of clinical case reports. Journal of the Medical Library

Association, v. 104, n. 4, p. 325-327, out. 2016. Disponível em: https://www.ncbi.nlm.nih.gov/pmc/articles/PMC5079498/. Acesso em: 20 fev. 2019.

NAVES, M. M. L. Estudo de fatores interferentes no processo de análise de assunto. Perspectivas em Ciência da Informação, v. 6, n. 2, p. 189-203, jul./dez. 2001. Disponível em:

http://portaldeperiodicos.eci.ufmg.br/index.php/pci/article/view/428. Acesso em: 20 abr. 2019.

NATIONAL INSTITUTES OF HEALTH. NLM administrative supplements for informationist services in NIH-funded research projects, 2011. Disponível em: https://grants.nih.gov/grants/guide/pa-files/PA-12-158.html. Acesso em: 20 abr. 2019.

NATIONAL INSTITUTES OF HEALTH. Data Services, 2019a. Disponível em: https://www.nihlibrary.nih.gov/services/data. Acesso em: 20 abr. 2019.

NATIONAL INSTITUTES OF HEALTH. Informationist Program, 2019b. Disponível em: https://www.nihlibrary.nih.gov/services/informationists. Acesso em: 07 abr. 2019.

PAI, M.; McCULLOCH, M.; GORMAN, J. D.; PAI, N.; ENANORIA, W.; KENNEDY, G.; THARYAN, P.; COLFORD, J. M. Systematic Reviews and Meta-Analyses: An Illustrated, Step-by-Step Guide. National Medical Journal of India, v. 17, n. 2, p. 89-95, 2004. Disponível em:

https://read.qxmd.com/read/15141602/systematic-reviews-and-meta-analysesan-illustrated-step-by-step-guide. Acesso em: 15 nov. 2018.

PRUDENCIO, D. S. Diretrizes curriculares nacionais e a construção de propostas curriculares inovadoras: um estudo de cotejamento dos projetos políticos pedagógicos da UNIRIO e UFMG. 2015. Dissertação (Mestrado em Ciências da Informação). Universidade Federal Fluminense, Niterói, 2015. Disponível em: https://app.uff.br/riuff/handle/1/7528. Acesso em: 24 ago. 2019. 
RANKIN, J. A.; GREFSHEIM, S. F.; CANTO, C. C. The emerging informationist specialty: a systematic review of the literature. Journal of the Medical Library Association: JMLA, [s.I.], v. 96, n. 3, p.194-206, jul. 2008. Disponível: http://dx.doi.org/10.3163/1536-5050.96.3.005. Acesso em: 20 mar. 2019.

ROBISON, R. R. Informationist Education. Medical Reference Services Quarterly, [s.I.], v. 27, n. 3, p. 339-347, aug. 2008. Disponível em: http://dx.doi.org/10.1080/02763860802199034. Acesso em: 02 jun. 2020.

SLADEK, R. M.; PINNOCK, C.; PHILLIPS, P. A. The informationist: a prospective uncontrolled study. International Journal for Quality in Health Care, Oxford, v. 16, n. 6, p. 509-515, dec. 2004. Disponível em: http://dx.doi.org/10.1093/intqhc/mzh084. Acesso em: 10 jun. 2019.

SHIPMAN, J.; HOMAN, M. Medicine's library lifeline. Library Journal, New YORK, v. 128, n. 6, p.49-50, abr. 2003. Disponível em: https://eric.ed.gov/?id=EJ673360. Acesso em: 20 fev. 2019.

SILVA, F. C. C. O papel dos bibliotecários na gestão de dados científicos. Revista Digital de Biblioteconomia \& Ciência da Informação, v. 14, n. 3, p. 387-406, 2016. Disponível em: https://periodicos.sbu.unicamp.br/ojs/index.php/rdbci/article/view/8646333. Acesso em: 07 jun. 2019.

TENOPIR, C.; BIRCH, B.; ALLARD, S. Academic libraries and research data services: current practices and plans for the future. Chicago, IL: Association of College and Research Libraries, jun. 2012. Disponível em:

http://www.ala.org/acrl/sites/ala.org.acrl/files/content/publications/whitepapers/T enopir_Birch_Allard.pdf. Acesso em: 14 maio 2019.

TRAVASSOS, G. H.; SANTOS, P. S. M.; NETO, P. G. M.; BIOLCHINI, J. An Environment to Support Large Scale Experimentation in Software Engineering. In: INTERNATIONAL CONFERENCE ON ENGINEERING OF COMPLEX COMPUTER SYSTEMS, 13., Anais [...], 2008. Northern Ireland. Belfast, 2008. Disponível em: https://ieeexplore.ieee.org/document/4492892. Acesso em: 24 mar. 2019.

VALENTIM, M. L. P. Formação: competências e habilidades do profissional da informação. In: VALENTIM, M. L. P. Formação do profissional da informação. São Paulo: Polis, 2002. p. 117-132.

WENGER, E. Communities of Practice: Learning, Meaning, and Identity. New York: Cambridge University Press, 1999.

ZABALA, A.; ARNAU, L. Como aprender e ensinar competências. Porto Alegre: Artmed, 2010. 


\title{
RESEARCH INFORMATIONIST: OPPORTUNITIES FOR LIBRARIANS IN THE BIG DATA ERA
}

\begin{abstract}
Introduction: it presents opportunities for the health sciences librarian to act as a research informationist, in response to the new requirements for the dissemination of research data required by funding agencies and scientific publishers. Objective: the main objective is the debate on how this professional can contribute to the development of strategies for searching and disseminating data in the Health Sciences area in the Brazilian context. Methodology: it uses a quasi-systematic review as a research method to present a historical overview of occupation, examine the professional profile in terms of skills and abilities needed to work in the world of work and illustrates some professional experiences documented in the international literature that suggest a trail of good practices that can be applied to the national context. This is an exploratory-descriptive research with a qualitative approach to data analysis and demonstration of results. Results: it concludes that these professionals act as mediators between clinical teams and specialized and updated information. In addition, they operate at all stages of the research process, providing guidance on formats, publication sources, copyright provisions, and advising on the entire editorial process. However, its main mission is related to improving research data management. Conclusions: it is concluded that the role of the research informationist allows the librarian to be recognized as a co-producer of health knowledge and to effectively fulfill more active roles in scientific development.
\end{abstract}

Descriptors: Librarian. Research Informationist. Infomationist. Data Management.

\section{INFORMACIONALISTA DE INVESTIGACIÓN: OPORTUNIDADES PARA LOS BIBLIOTECARIOS EN LA ERA DE LOS GRANDES DATOS.}

\begin{abstract}
RESUMEN
Introducción: presenta oportunidades para que el bibliotecario de ciencias de la salud actúe como informador de investigación, en respuesta a los nuevos requisitos para la difusión de datos de investigación requeridos por agencias de financiación y editoriales científicas. Objetivo: el objetivo principal es el debate sobre cómo este profesional puede contribuir al desarrollo de estrategias para buscar y diseminar datos en el área de Ciencias de la Salud en el contexto brasileño. Metodología: utiliza una revisión cuasi sistemática como método de investigación para presentar una descripción histórica de la ocupación, examinar el perfil profesional en términos de habilidades y destrezas requeridas para trabajar en el mundo laboral e ilustra algunas experiencias profesionales documentadas en la literatura internacional que sugieren un rastro de buena prácticas que pueden aplicarse al contexto nacional. Esta es una investigación exploratoria descriptiva con un enfoque cualitativo para el análisis de datos y la demostración de resultados. Resultados: comprobamos que estos profesionales actúan como mediadores entre equipos clínicos e información especializada y actualizada. Además, operan en todas las etapas del proceso de investigación, proporcionando orientación sobre formatos, fuentes de publicación, disposiciones de
\end{abstract}

Inf. Inf., Londrina, v. 25, n. 2, p. 150 - 177, abr./jun. 2020. 
derechos de autor y asesorando sobre todo el proceso editorial. Sin embargo, su misión principal está relacionada con la mejora de la gestión de datos de investigación. Conclusiones: se concluye que el rol del informador de investigación permite que el bibliotecario sea reconocido como coproductor del conocimiento de la salud y que cumpla efectivamente roles más activos en el desarrollo científico.

Descriptores: Bibliotecario. Informacionista. Gestión de datos. Profesional de la información.

Recebido em: 07.10.2019

Aceito em: 06.12.2019 